\title{
Tratamiento ortodóntico interceptivo para paciente pediátrico con problema periodontal ocasionado por trauma oclusal primario. Reporte de caso
}

Johana Maldonado-Villamizar ${ }^{1}$

\section{Resumen}

El diagnóstico correcto puede evitar la evolución de patologías que lleven al paciente pediátrico a padecer de problemas graves en el futuro. Este diagnóstico junto con un tratamiento interceptivo a tiempo, puede ayudar a resolver problemas de tipo periodontal causado por el inadecuado posicionamiento dental o injurias oclusales. El objetivo de este artículo es realizar el reporte de caso de un paciente pediátrico que presenta una maloclusion dental tipo mordida cruzada anterior acompaña- da por un trauma oclusal primario, el cual causo un problema periodontal localizado, a su vez, describir la terapéutica interceptiva basada en el correcto diagnóstico odontopediátrico, ortodóntico y periodontal. Concluyendo que al seleccionar la aparatología adecuada se puede solventar diversos problemas dentales que puedan ocasionar un daño mayor a las estructuras comprometidas.

Palabras Claves: Ortodoncia, interceptivo, periodontal, trauma oclusal, recesión gingival, biteblock.

Relato de caso

\section{Tratamento ortodôntico interceptivo para paciente pediatrico con problema periodontal ocasionado por trauma oclusal primário. Relato de caso}

\section{Resumo}

O diagnóstico correcto pode prevenir a evolução de doenças que conduzem a pacientes pediátricos a desenvolver sérios problemas no futuro. Este diagnóstico, juntamente com o tratamento interceptivo a tempo, pode ajudar a resolver problemas do tipo periodontal causada pelo posicionamento dental incorreto ou problemas oclusais. O objetivo deste artigo é relatar um caso de um paciente pediátrico que apresenta maloclusáo tipo mordida cruzada anterior acompanhada por um trauma oclusal primário, o qual causou um problema periodontal localizado, e, por ocasião disso,er a terapia interceptiva baseada no correcto diagnóstico odontopediátrico, ortodôntico e periodontal. Conclui-se que selecionar a ferramenta certa pode-se resolver muitos problemas dentários que poderiam causar danos maiores às estruturas comprometidas

Palabras Claves: ortodontia, interceptativo, periodontal, trauma oclusal, recessão gengival, bloco de mordida. 


\section{Interceptive orthodontic treatment for pediatric patient with periodontal problems caused by occlusal trauma primary. Case report}

\begin{abstract}
Correct diagnosis can prevent the development of diseases that lead to pediatric patients to develop serious problems in the future. This diagnosis with early interceptive treatment, can help solve problems of periodontal kind caused by improper positioning or occlusal dental injuries. The aim of this paper is to report a case of a pediatric patient with a dental malocclusion type crossbite accompanied
\end{abstract}

\section{Introducción:}

Los tejidos de la cavidad oral están estrechamente interrelacionados, por lo tanto, cuando alguno de ellos presenta una patología, el resto se puede afectar en grado variable dependiendo de su relación funcional y de proximidad física. ${ }^{1-2}$ Debido a esto, la presencia de una malposición dentaria es considerada uno de los factores de riesgo frecuentemente asociado a un problema periodontal en el paciente pediátrico; ya que cuando existe una malposición la ubicación del margen gingival se encuentra alterada, la textura de la encía varía, hay tendencia a una lámina delgada del alveolo y menor cantidad de encía insertada que favorece el desarrollo de recesión gingival. ${ }^{3}$

Una de las malposiciones o maloclusiones dentales que causan y/o se asocian a un problema periodontal son las mordidas cruzadas anteriores, las cuales pueden ser producidas por inclinación inadecuada de los incisivos maxilares y mandibulares, por interferencias oclusales (funcionales) o por discrepancias esqueléticas del maxilar y/o mandíbula. ${ }^{3-4}$ Debido a estas situaciones se produce un trauma oclusal primario, el cual es el daño de los tejidos provocado alrededor de un diente con periodonto de altura normal como resultado de fuerzas excesivas. Este trauma sobre un by a primary occlusal trauma, which caused a localized periodontal problem, in turn, describe the interceptive therapy based correct pediatric dental, orthodontic and periodontal diagnosis. Concluding that selecting the right appliance can solve many dental problems that could cause further damage to the compromised structures.

Key words: Orthodontics, interceptive, periodontal, occlusal trauma, gingival recession, biteblock.

periodonto sano con altura normal produce un aumento de la movilidad por un ensanchamiento del ligamento periodontal, que deja de aumentar cuando el diente se adapta a la situación. ${ }^{5}$

Una alternativa ortodóntico terapéutica para generar un cambio oclusal en estos casos de mordida cruzada anterior es el bite block, debido a que su acción biomecánica y principio de acción está basado en una fuerza intermitente de los músculos elevadores de la mandíbula ajustando la dimensión vertical para el posterior descruce de la mordida anterior ${ }^{6-7-8}$ eliminando de esta manera el trauma oclusal y mejorando la situación periodontal.

Por lo anteriormente expuesto el objetivo de este artículo es reportar una alternativa de tratamiento ortodóntico interceptivo para paciente pediátrico con problema periodontal ocasionado por trauma oclusal primario.

\section{Reporte de Caso}

Se presenta paciente masculino de 10 años de edad, procedente de Valencia - Estado Carabobo, que al referir el motivo de consulta el representante expone "Arreglarle los dientes". Entre los 


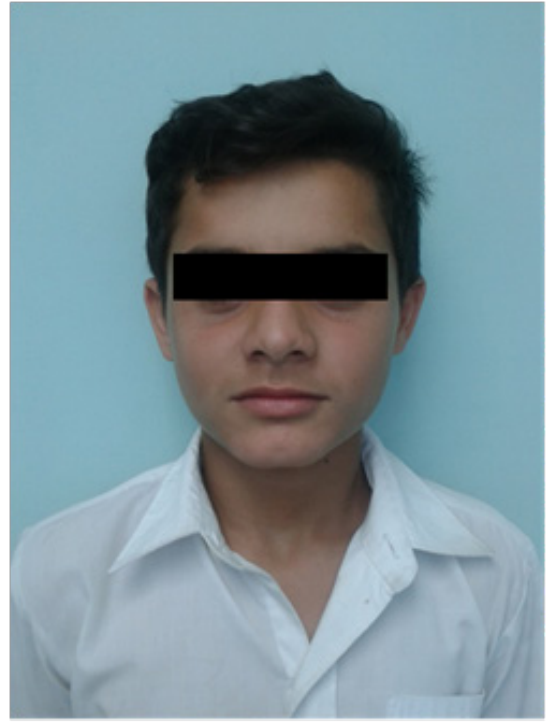

Foto 1-A

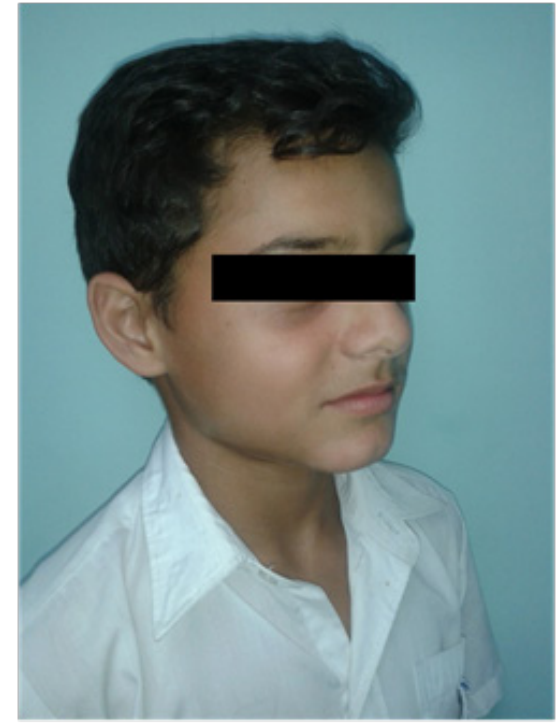

Foto 1-B

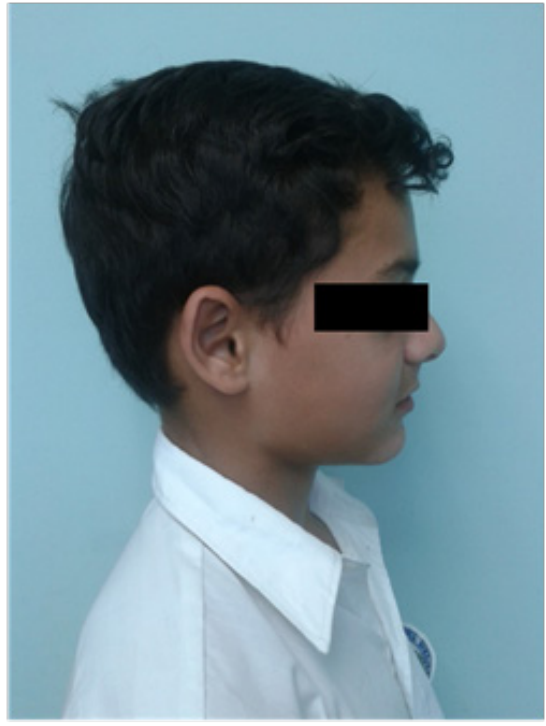

Foto $1-\mathrm{C}$

Figura 1. Fotografías Extraorales Iniciales.

Foto 1-A Fotografía de frente. Foto 1-B Fotografía 3/4 derecha. Foto 1-C Fotografía perfil derecho.

antecedentes personales refiere poseer patología tipo hipertrofia adenoidea diagnosticada por el otorrinolaringólogo y extirpada quirúrgicamente a los 8 años. De igual manera, refiere que su representado sufrió un traumatismo en cara a los 6 años de edad que involucro trauma en estructuras dentales anteriores. A nivel dental el paciente denota que presenta sensibilidad en unidades dentales (UD) 31 y 41 . La representante acota que no ha sido tratado ortodontica - ortopédicamente.

Bajo consentimiento informado, el representante permitió el uso de toda la información que corresponda a la historia clínica, diagnósticos, estudios complementarios, fotografías de seguimiento, tratamiento finalizado, las cuales fueron utilizados de manera profesional y científica, preservando el anonimato del representado.

Análisis Extraoral y Fotográfico: Inserción adecuada del cabello y orejas, ojeras, cara ovalada, ligera asimetría facial, tercio inferior disminuido, perfil recto, depresión hueso malar del tercio medio de la cara, surco labial superior recto, posicionamiento adecuado del mentón (Figura $\mathrm{N}^{\circ} 1$ ).

Análisis Intraoral: clase I molar derecha, clase III molar izquierda, clase I canina derecha, clase III canina izquierda, línea media inferior desviada derecha $3 \mathrm{~mm}$, mordida cruzada anterior localizada en UD 11 y 21, extrusión del UD 11, rotación de UD 12,22,32 y 42 overbite aumentado compatible

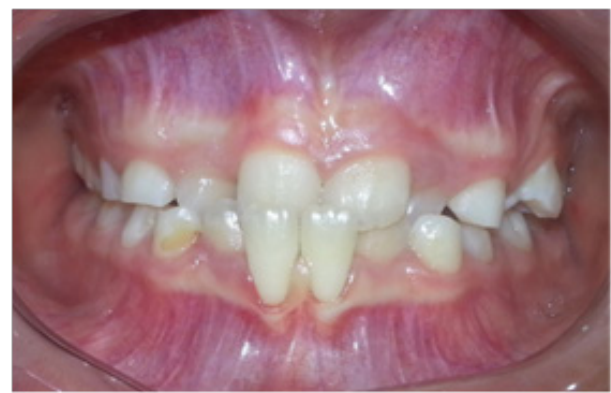

Foto $2-A$

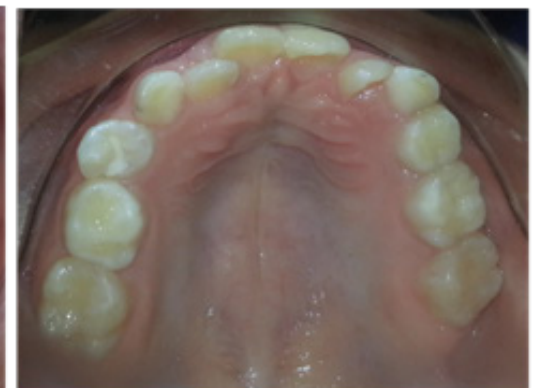

Foto $2-B$

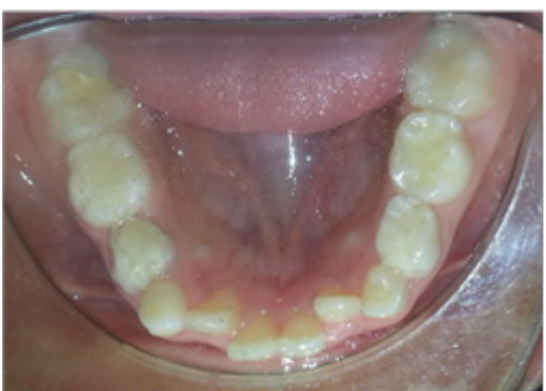

Foto $2-\mathrm{C}$

Figura 2. Fotografías Intraorales Iniciales.

Foto 2-A Fotografía de frente. Foto 2-B Fotografía oclusal superior. Foto 2-C Fotografía oclusal inferior. 


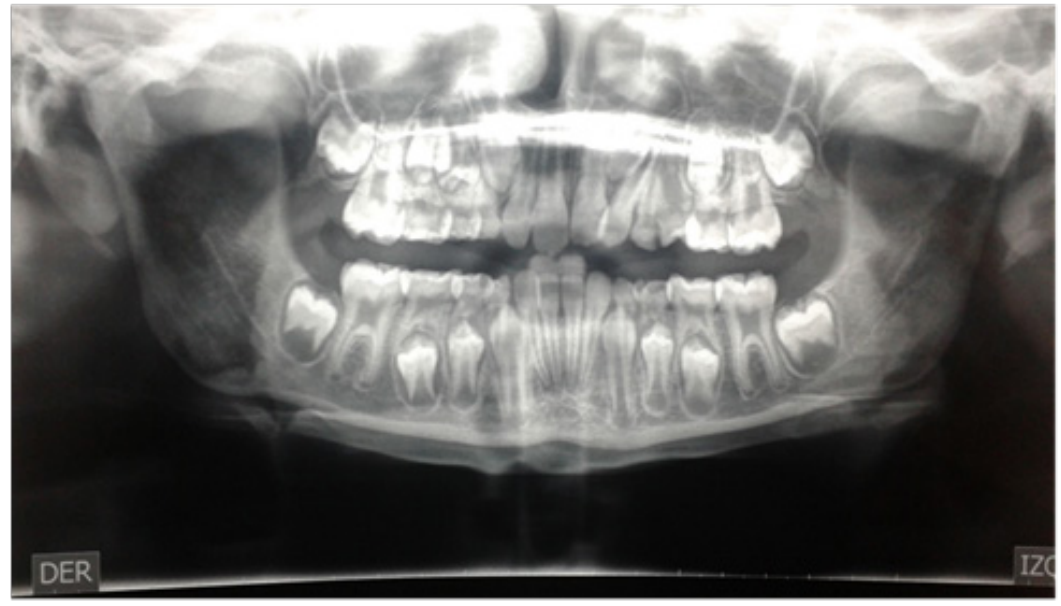

Foto 3-A

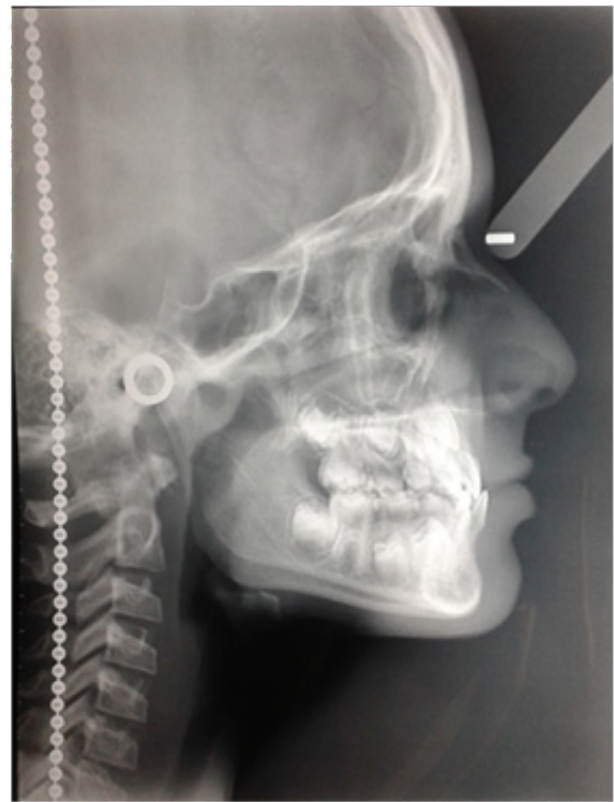

Foto 3-B

Figura 3. Estudios Radiológicos Iniciales.

con mordida profunda, mordida telescópica localizada UD 24, curva spee plana. Periodontalmente muestra una fenestración en el hueso alveolar y recesión periodontal clase I en UD 31 y 41 con un biotipo periodontal delgado, movilidad grado II en UD 31 y 41 acompañadas por trauma oclusal primario (Figura $\mathrm{N}^{\circ} 2$ ). Al referir al odontopediatra, esta genera como diagnóstico disminución del nivel óseo, y ensanchamiento del ligamento periodontal localizado en UD 31 y 41.

Análisis de Modelos: Con respecto a la forma de arco se observa que el maxilar superior presenta una forma redonda y el inferior una forma triangular. Al realizar las mediciones de las distancias intercanina $(33 \mathrm{~mm})$ e intermolar $(52 \mathrm{~mm})$ se observa una sobre expansión maxilar. Se evidencia discrepancia negativa en el análisis predictivo de Moyers en las sumas de ambas hemiarcadas, para el maxilar superior $-7 \mathrm{~mm}$ y para el maxilar inferior es de $-8 \mathrm{~mm}$.

Análisis Panorámico: Se observa UD 23 en un posicionamiento inadecuado con posible impactación y reabsorción de raíz radicular de UD 22. Inclinación de tipo tip derecho de la UD 22 (Figura $\mathrm{N}^{\circ} 3$ ).

\section{Análisis Cefalométrico:}

Posicionamiento maxilar: Paciente presenta una retrusión maxilar, diagnosticada a partir del ángulo SNA $77^{\circ}, \mathrm{N}$ - Perpendicular a Maxilar - 3mm y corroborada por la profundidad maxilar de $87^{\circ}$. Posicionamiento mandibular: Posición normal para la edad del paciente SNB $78^{\circ}, \mathrm{N}$ - Perpendicular a mandíbula de $-3 \mathrm{~mm}$ y corroborada con la profundidad facial de $89^{\circ}$, valorando que el paciente se encuentra en crecimiento y al momento del pico de crecimiento los ángulos se proyectan aproximadamente $3^{\circ}$, lo cual permite mantenerse dentro de la norma de crecimiento anteroposterior mandibular. Con respecto a la longitud mandibular se encuentra dentro de la norma en proporción 1: 1 de Jarabak con respecto a la longitud de la base craneal y la longitud mandibular es de $76 \mathrm{~mm}$ cada una, afirmando un crecimiento adecuado mandibular.

Clase Esquelética y Biotipo Facial: El paciente presenta una clase III esquelética arrojada por el ángulo ANB $-1^{\circ}$ y la convexidad de Ricketts de $-2 \mathrm{~mm}$. Su biotipo es braquifacial severo, diagnosticado a partir del Vert de Ricketts y afirmado con ángulos SN- SGN 52 ${ }^{\circ} \mathrm{Pl} \mathrm{Mn}-\mathrm{Fh} 18^{\circ}, \mathrm{SN}-\mathrm{Pl}$ 
Mn $28^{\circ}$. La deflexión mandibular generada por el ángulo góniaco total de Jarabak es de $123^{\circ}$, lo que indica una rotación mandibular en contra de las agujas del reloj. La proporción facial de Jarabak se encuentra entre el 67, 28\% (Braquifacial Severo).

Posiciones e Inclinaciones Dentales: Los ángulos que evalúan al incisivo superior arrojan que se encuentra con una adecuada inclinación dental, los valores arrojados fueron los siguientes: 1- NS $103^{\circ}, 1 \mathrm{~A}-\operatorname{pog} 25^{\circ}, 1 \mathrm{~N}-\mathrm{A} 24^{\circ}$. Ahora bien, a nivel de posicionamiento del incisivo se muestra una retrusión dental, valores como $1 \mathrm{~N}-\mathrm{A} \mathrm{mm}-1 \mathrm{~mm}$, 1 A-pog -2mm. Ahora bien, el incisivo inferior se encuentra proinclinado mostrado por los ángulos 1 -Pl Mn 97, 1 A- Pog 25, a nivel de posicionamiento se encuentra dentro de la norma con un 1 - A Pog 2mm.

Posición de los Labios: La Línea E de Ricketts muestra una retrusión del labio superior y un buen posicionamiento del labio inferior.

\section{Resumen Cefalométrico:}

Paciente masculino de 10 años de edad que presenta una clase III esquelética por retrusión maxilar, un biotipo braquifacial. A nivel dental presenta una retrusión del incisivo superior y proinclinación del incisivo inferior. En tejidos blando presenta una retrusión del labio superior (Figura $N^{\circ} 4$ ).

\section{Plan de Tratamiento}

Es común en el consultorio odontopediátrico el reporte de mordida cruzadas anteriores en dentición mixta, pero poca es la prevalencia de aquellas acompañadas por problemas esqueléticos y periodontales. La solución de todas las situaciones diagnósticas en una sola fase, se considera ideal para que en el paciente odontopediátrico se observen cambios favorables en poco tiempo de ejecución de la mecánica. Por esta razón el plan de tratamiento indicado para este caso en particular fue el siguiente:

\section{Fase I: Bite Block con doble coffen y tracción con máscara facial.}

El plan de tratamiento fue establecido por fases, en este artículo se desglosará la fase I interceptiva basada en la colocación de un bite block cementado con la finalidad de levantar la mordida para realizar el descruce de las UD 31 y 41, y a su vez, eliminar el trauma oclusal primario disminuyendo la injuria oclusal y la movilidad dental. Este bite block se presenta un doble coffen, se evitó la colocación de tornillo de expansión, ya que el maxilar presenta una sobrexpansión confirmada con las distancias intercanina e intermolar. Adicional al biteblock se coloca ganchos para tracción con mascara extraoral. Se contraindica la colocación de resortes palatinos debido a que la inclinación dentoalveolar es adecuada (1- NS 103, 1 A-pog $\left.25^{\circ}, 1 \mathrm{~N}-\mathrm{A} 24^{\circ}\right)$, la colocación de estos aditamentos

\begin{tabular}{|c|c|c|c|}
\hline Estructura Anatómica Evaluada & Steiner Inicial & $\begin{array}{c}\text { Mc Namara / Jarabak } \\
\text { Inicial }\end{array}$ & $\begin{array}{l}\text { Ricketts } \\
\text { Inicial }\end{array}$ \\
\hline Posición antero-posterior maxilar & $77^{\circ}$ & $-3 \mathrm{~mm}$ & $87^{\circ}$ \\
\hline Posición antero-posterior mandíbula & $78^{\circ}$ & $-3 \mathrm{~mm}$ & $89^{\circ}$ \\
\hline Longitud Mandibular & - & $76 \mathrm{~mm}$ & $65 \mathrm{~mm}$ \\
\hline Posición del incisivo superior & $\begin{array}{c}1 \mathrm{~N}-\mathrm{A} \mathrm{mm}-1 \\
\mathrm{~mm}\end{array}$ & - & 1 A-pog $-2 \mathrm{~mm}$ \\
\hline Posición del incisivo inferior & $1 \mathrm{~N}-\mathrm{B} \mathrm{mm} 2 \mathrm{~mm}$ & - & 1 A-pog $-2 \mathrm{~mm}$ \\
\hline Inclinación del incisivo superior & $\begin{array}{l}1-\mathrm{NS} 103^{\circ} \\
1 \mathrm{~N}-\mathrm{A} 24^{\circ}\end{array}$ & - & $1 \mathrm{~A}-\operatorname{pog} 26^{\circ}$ \\
\hline Inclinación del incisivo inferior & 1 -PI Mn 970 & - & $1 \mathrm{~A}-\operatorname{pog} 25^{\circ}$ \\
\hline
\end{tabular}

Figura 4. Medidas cefalométricas iniciales según estructura anatómica evaluada. 


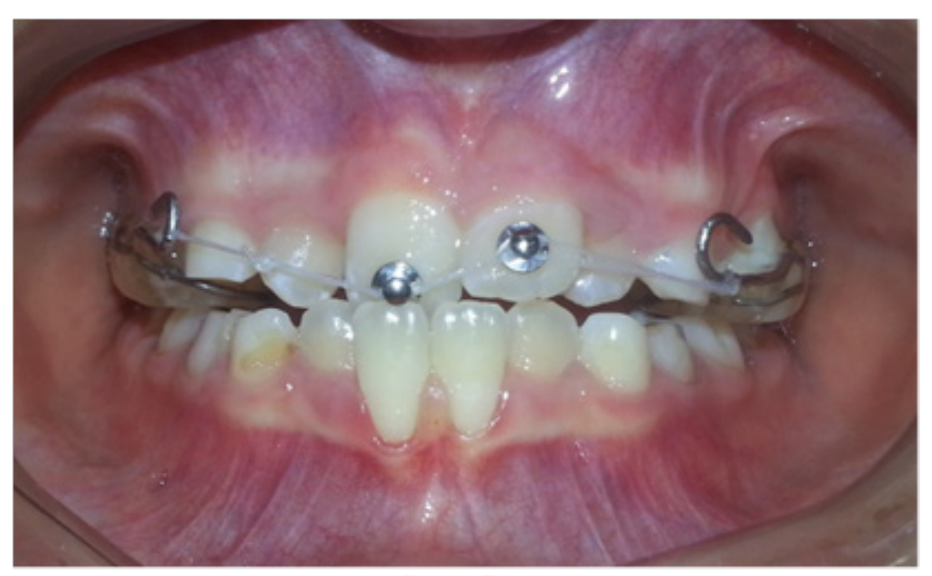

Foto 5-A

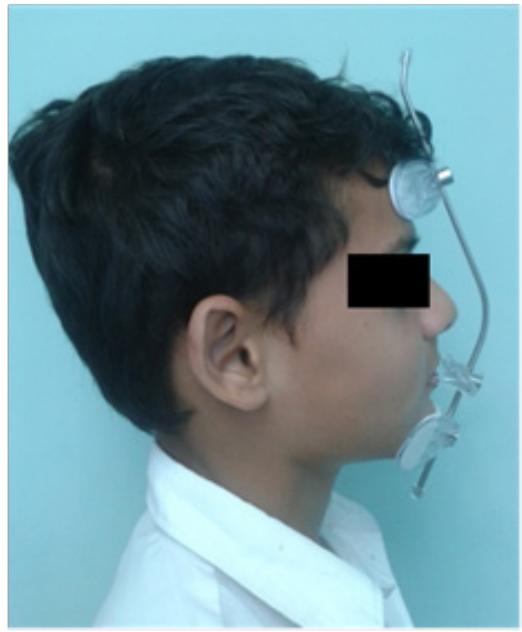

Foto $5-\mathrm{C}$

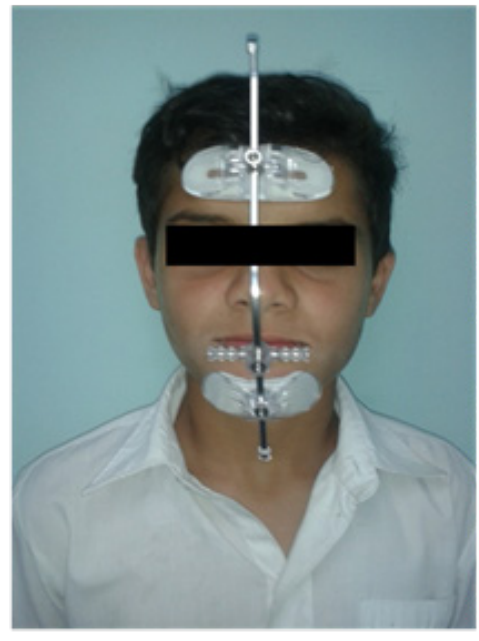

Foto $5-\mathrm{D}$

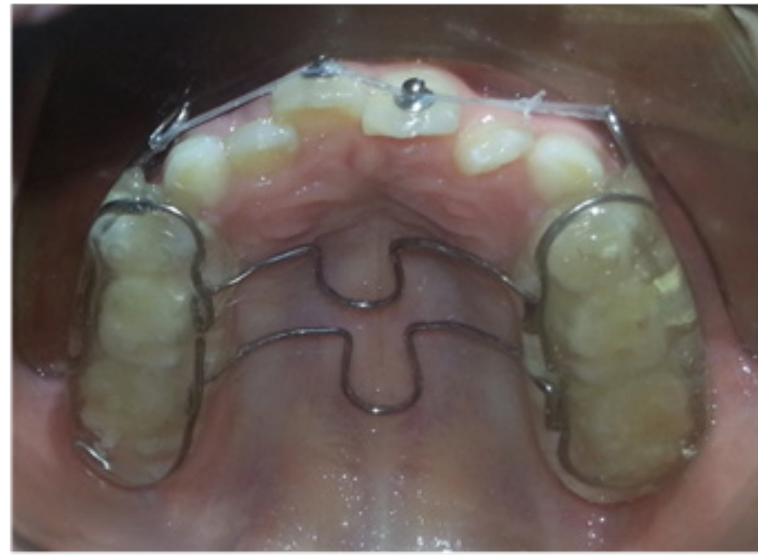

Foto 5 -B

Figura 5. Fotografías de Tratamiento Interceptivo.

Foto 5-A Fotografía de frente intraoral con bite block. Foto 5-B Fotografía oclusal superior con bite block.

Foto 5-C Fotografía extraoral de frente con máscara facial.

Foto 5-D Fotografía extraoral de perfil con máscara facial.

de protrusión, ocasionaría inclinaciones incisales inadecuadas.

Para la tracción extraoral se colocó una máscara de Petti con elásticos extraorales bilaterales de $3 / 8^{\prime \prime}$ y 8 onzas, sujetados de ganchos intraorales colocados a nivel de canino. La tracción debe realizarse por lo menos 12 horas continuas y se recomendó que fueran en horas de la noche por la segregación somatotropina (hormona del crecimiento). La colocación de la máscara facial facilitará la tracción del maxilar superior, y a su vez, mejorará la posición de retrusión del incisivo superior. Se colocó botones ortodónticos en la UD 11 y 21 y a partir de los ganchos para la tracción extraoral se colocó un hilo ortodóntico para la intrusión de la UD 11(Figura $\mathrm{N}^{\circ} 5$, específicamente en figura 5-A, 5-B). Se contraindica la colocación de brackets en UD 12,21,22; debido al eje de inclinación de las UD 13 y 23, en parti- cular, la ubicación del 23, el cual bidimensionalmente se observa desplazando la raíz de UD 22 y cercanía de la corona del 23 a la raíz del 21. Un movimiento ortodóntico a partir de brackets en UD 12, 21,22; traería consigo proceso de reabsorción radicular en dichas estructuras.

Se realiza la valoración mensual del paciente donde se observa la salud periodontal del paciente y el nivel de tracción extraoral (corroborado con un dinamómetro), de igual manera, pasados 2 meses se envía la realización de una cefálica lateral y se evalúa con la cefalometría de Steiner y Ricketts, observando un SNA $82^{\circ}$, Profundidad Maxilar $92^{\circ}$, lo que corresponde una posición anteroposterior adecuado del maxilar superior, dentalmente se observa una posición adecuada del incisivo superior, esto debido a que al mejorar la retrusión maxilar, mejora la retrusión dentoalveolar superior y los ángulos $1 \mathrm{~N}$-A mm y $1 \mathrm{~A}$-pog, ya que 


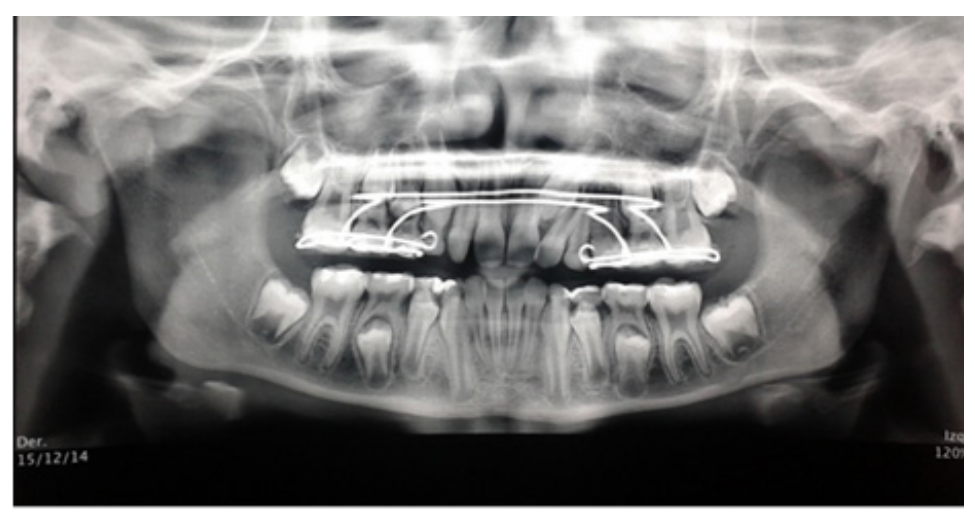

Foto 6-A

Figura 6. Estudios Radiográficos Finales para Fase Interceptiva. Foto 6-A Radiografía panorámica.

Foto 6-B Radiografía cefálica lateral.

los mismo dependen de la posición intermaxilar; adicionando que las fuerzas reciprocas de ambos incisivos generaron la alineación deseada (Figura $N^{\circ} 6$ y 7). Se continua con la utilización de la máscara 2 meses más como periodo de retención.

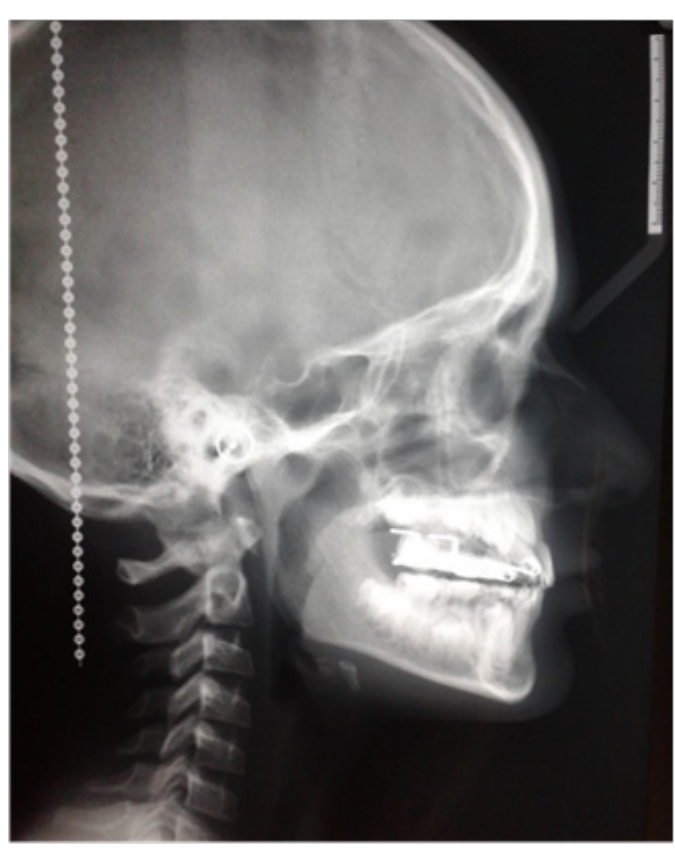

Foto 6-B
Posterior a 4 meses de tracción extraoral e intrusión dental se observa posicionamiento adecuado del maxilar superior, descruce dental de las UD 11 y 21, intrusión de la UD 11, eliminación de la movilidad dental y continuidad

\begin{tabular}{|c|c|c|c|c|c|c|}
\hline \multirow[b]{2}{*}{ Estructura Evaluada } & \multicolumn{2}{|c|}{ Steiner } & \multicolumn{2}{|c|}{ Mc Namara / Jarabak } & \multicolumn{2}{|c|}{ Ricketts } \\
\hline & Steiner Inicial & Steiner Final & $\begin{array}{c}\text { Mc Namara } \\
\text { / Jarabak } \\
\text { Inicial }\end{array}$ & $\begin{array}{c}\text { Mc Namara } \\
\text { / Jarabak } \\
\text { Final }\end{array}$ & $\begin{array}{l}\text { Ricketts } \\
\text { Inicial }\end{array}$ & $\begin{array}{l}\text { Ricketts } \\
\text { Final }\end{array}$ \\
\hline $\begin{array}{c}\text { Posición } \\
\text { antero-posterior maxilar }\end{array}$ & $77^{\circ}$ & $81^{\circ}$ & $-3 \mathrm{~mm}$ & $1 \mathrm{~mm}$ & $87^{\circ}$ & $91^{\circ}$ \\
\hline $\begin{array}{c}\text { Posición } \\
\text { antero-posterior mandíbula }\end{array}$ & $78^{\circ}$ & $79^{\circ}$ & $-3 \mathrm{~mm}$ & - & $89^{\circ}$ & $88^{\circ}$ \\
\hline Longitud Mandibular & - & - & $76 \mathrm{~mm}$ & $77 \mathrm{~mm}$ & $65 \mathrm{~mm}$ & $65 \mathrm{~mm}$ \\
\hline $\begin{array}{c}\text { Posición } \\
\text { del incisivo superior }\end{array}$ & $\begin{array}{l}1 \mathrm{~N}-\mathrm{A} \mathrm{mm}-1 \\
\mathrm{~mm}\end{array}$ & $1 \mathrm{~N}-\mathrm{A} \mathrm{mm} 2 \mathrm{~mm}$ & - & - & $\begin{array}{l}1 \mathrm{~A}-\mathrm{Pog} \\
-2 \mathrm{~mm}\end{array}$ & $\begin{array}{l}1 \mathrm{~A}-\mathrm{Pog} \\
-1 \mathrm{~mm}\end{array}$ \\
\hline $\begin{array}{c}\text { Posición } \\
\text { del incisivo inferior }\end{array}$ & $1 \mathrm{~N}-\mathrm{B} \mathrm{mm} 2 \mathrm{~mm}$ & $1 \mathrm{~N}-\mathrm{B} \mathrm{mm} 2 \mathrm{~mm}$ & - & - & $\begin{array}{l}1 \mathrm{~A}-\mathrm{Pog} \\
-2 \mathrm{~mm}\end{array}$ & $\begin{array}{l}1 \mathrm{~A}-\mathrm{Pog} \\
-2 \mathrm{~mm}\end{array}$ \\
\hline $\begin{array}{c}\text { Inclinación } \\
\text { del incisivo superior }\end{array}$ & $\begin{array}{l}1 \text {-NS } 103^{\circ} \\
1 \mathrm{~N}-\mathrm{A} 24^{\circ}\end{array}$ & $\begin{array}{l}1 \text {-NS } 105^{\circ} \\
1 \mathrm{~N}-\mathrm{A} 25^{\circ}\end{array}$ & - & - & $\begin{array}{c}1 \text { A-Pog } \\
26^{\circ}\end{array}$ & $\begin{array}{c}1 \mathrm{~A}-\mathrm{Pog} \\
25^{\mathrm{o}}\end{array}$ \\
\hline $\begin{array}{c}\text { Inclinación } \\
\text { del incisivo inferior }\end{array}$ & 1- PI Mn 97º & 1- PI Mn 95 & - & - & $\begin{array}{l}1 \mathrm{~A}-\mathrm{Pog} \\
25^{\mathrm{o}}\end{array}$ & $\begin{array}{l}1 \mathrm{~A}-\mathrm{Pog} \\
26^{\circ}\end{array}$ \\
\hline $\begin{array}{l}\text { Proporciones Faciales } \\
\text { (Biotipo Facial) }\end{array}$ & $\begin{array}{l}\text { SN- SGN 52० } \\
\text { PI Mn-Fh } 18^{\circ} \\
\text { SN- PI Mn } 28^{\circ}\end{array}$ & $\begin{array}{l}\text { SN- SGN 52० } \\
\text { PI Mn-Fh } 20^{\circ} \\
\text { SN- PI Mn } 31^{\circ}\end{array}$ & $67,28 \%$ & $68,28 \%$ & $+1,06$ & $+1,12$ \\
\hline
\end{tabular}

Figura 6. Estudios Radiográficos Finales para Fase Interceptiva. 


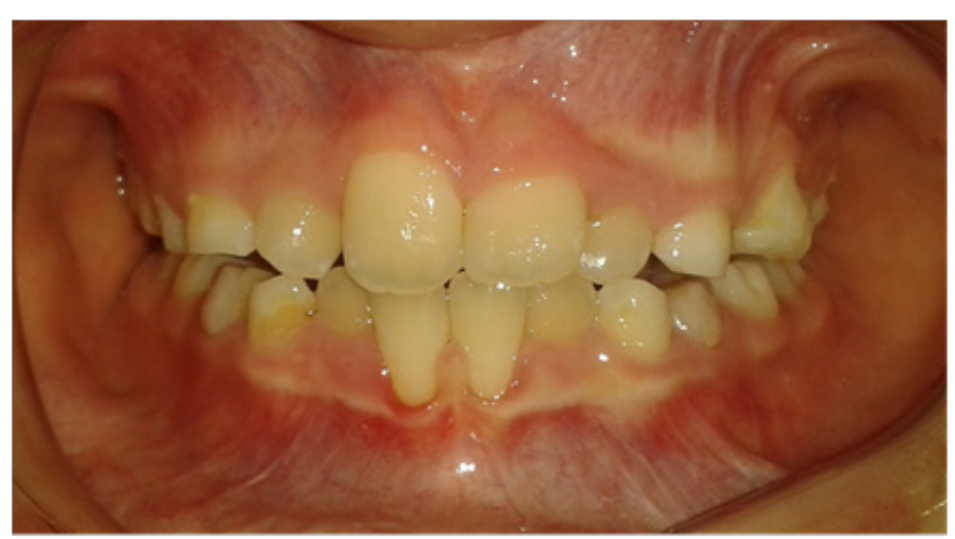

Foto 8-A

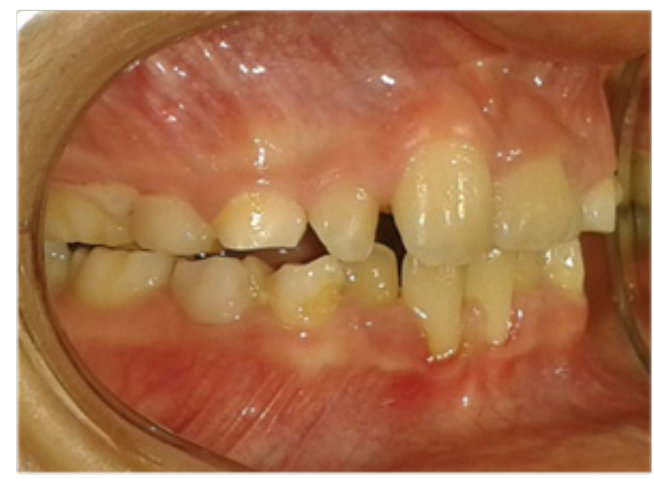

Foto 8-C

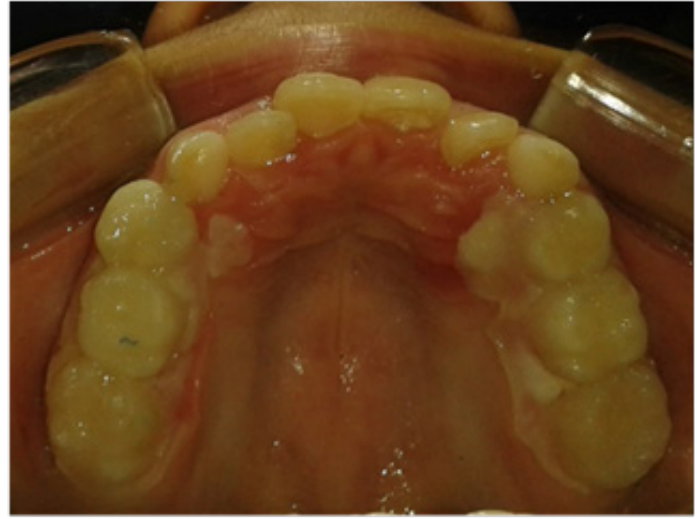

Foto 8-B

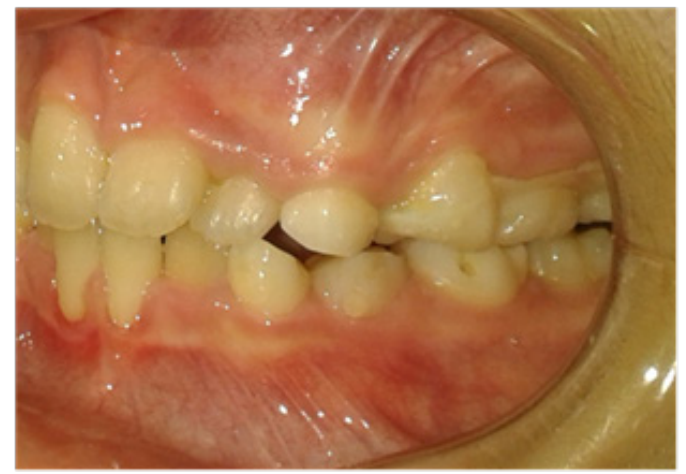

Foto 8-D

Figura 8. Fotografias Intraorales Finales (Fase I).

Foto 8-A Fotografía de frente. Foto 8-B Fotografía oclusal superior. Foto 8-C Fotografía lateral derecha.

Foto 8-D Fotografía lateral izquierda.

de recesión gingival dejadas por las secuelas del trauma oclusal primario en las UD 31 y 41 (Figura $\mathrm{N}^{\circ}$ 8). Se hace la referencia a Odontopediatría y periodoncista para su valoración y continuidad de siguientes fases de tratamiento.

\section{Discusión}

La cooperación interdisciplinaria y del paciente puede transformar los problemas dentales y periodontales, en sonrisas y dentaduras estéticamente saludables; siendo recomendable que el odontopediatra y el ortodoncista incorpore en el tratamiento una evaluación periodontal durante la consulta inicial. ${ }^{5}$

En el paciente odontopediátrico el tratamiento interceptivo de tipo ortodóntico y periodontal evita que en el futuro existan secuelas y pro- fundización de las patologías existentes. En la actualidad existen aparatologías que permiten tratar problemas como la mordida cruzada anterior utilizados por el odontopediatra y el ortodoncista, un ejemplo de estos es el plano inclinado. En presencia de problemas periodontales causados por esta patología, la terapéutica tipo plano inclinado está contraindicada. ${ }^{4,9-10}$ De igual manera, la utilización de aparatología removible en estos casos de mordida cruzada anterior complicada con problemas periodontales causados por trauma oclusal primario, no sería una alternativa eficaz debido a que existen diferentes factores que conllevaría al fracaso. ${ }^{11}$

El uso del Bite Block funge como una alternativa efectiva en el tratamiento de las mordidas cruzadas anteriores presentando como ventaja ir cementado por lo que no se requiere la colaboración del paciente, se garantiza su uso y el 
objetivo del tratamiento es ejecutado en menor tiempo. ${ }^{8}$ Esta aparatología cementada permite la desoclusión del sector anterior eliminando el factor causal de la movilidad dental y el trauma primario generado en los incisivos inferiores, ${ }^{13-14}$ otra de la preeminencia que tiene este tipo de opción.

La utilización de máscara facial y aditamentos como los botones ortodónticos surgen como adicionales al tratamiento, los cuales van atacar otras necesidades de terapéutica que el paciente necesita, implementando así un tratamiento multifuncional en una sola fase de ejecución. ${ }^{14,15-16}$

\section{Conclusión}

El diagnóstico y la planificación inicial del tratamiento debe ser minuciosa, permitiendo que la ejecución del mismo permita mantener las estructuras que se encuentran en equilibrio natural no se vean afectadas por la terapéutica planteada. El uso de aparatología interceptiva para evitar el desencadenamiento de una patología más complicada de tratar en un futuro, es sin lugar a duda la visión del odontopediatra y el ortodoncista/ ortopedista, por esta razón se brinda una nueva opción para casos que presenten complicaciones ortoperiodontales como lo es el bite block con aditamentos adicionales.

\section{Referencias bibliográficas.}

1.- Tortolini P, Fernández Bodereau E. Ortodoncia y periodoncia. Av. Odontoestomatol 2011; 27 (4): 197-206.

2.- Hernández-Banda, Jazmin, and Alicia Gallegos-Ramírez. "Tratamiento ortopédico de síndrome prognatico, con mascara de protraccion." Consejo Editorial 21.1 (2015): 26-30.

3.- . Bracho R, Hernández N, Elejalde L, Zambrano O, Paz M, Contreras J. Recesión gingival de incisivos inferiores en adolescentes, su asociación con factores de riesgo. Acta Odontológica de Venezuela 2003; 41 (3).

4.- Herrera L, Maldonado J, Velásquez N, Pinto J. Mordida cruzada anterior: tratamiento ortodóntico interceptivo. Hass inverso. Reporte de un caso. Revista Latinoamericana de Ortodoncia y Odontopediatría 2015. Disponible en http://www. ortodoncia.ws/publicaciones/2015/ art6.asp

5.- Velázquez N, Valdivia I. Tratamiento ortodóncico en paciente clase III, periodontalmente comprometida: reporte de un caso. Revista Mexicana de Ortodoncia 2015;3 (4): 274-282

6.- Cuba, Viviana Salazar. "Manejo del trauma en pediatría Management of pediatric trauma." Revista de la Sociedad Boliviana de Pediatría 51.1 (2015).

7.- Sanz-Sánchez I., Bascones-Martínez A. Otras enfermedades periodontales: II: Lesiones endo-periodontales y condiciones y/o deformidades del desarrollo o adquiridas. Avances en Periodoncia. 2008; 20(1): 67-77.

8.- Segovia L, Peña E, Moreno O, Maldonado J. Uso del Bite Block en el tratamiento de Mordida Cruzada anterior en dentición primaria y mixta. Reporte de casos. Revista Latinoamericana de Ortodoncia y Odontopediatría 2015. Disponible en http://www.ortodoncia.ws/publicaciones/2015/art12.asp

9.- Segovia L, Maldonado J, Moreno O. Ortodoncia interceptiva aplicada al crecimiento de un paciente con Mordida Cruzada Anterior. Revista Latinoamericana de Ortodoncia y Odontopediatría 2015.Disponible en http://www.ortodoncia. ws/publicaciones/2015/art15.asp

10.- Quirós, O. El plano inclinado para el tratamiento de la mordida cruzada anterior. Acta Odontológica de Venezuela 2002; 40 (3).

11.- Villa D, Gasca G. Uso de coronas pediátricas invertidas y aparato fijo para la corrección de mordida cruzada anterior en dentición temporal. Revista ADM. 2011; 3: 132-5.

12.- Velásquez, V. Mordida cruzada anterior: Diagnóstico y tratamiento con Placa Progenie. Revista Latinoamericana de Ortodoncia y Odontopediatría 2005. Disponible en URL: http://www.ortodoncia.ws/publicaciones/2005/art8.asp

13.- Jorquera R, Leiva N. Tratamiento periodontal y ortodóncico de un caso de periodontitis agresiva , 12 años de seguimiento. Braz J Periodontol 2011; 21(4):55-59.

14.- Escalona E, Ruiz M, Rivera H, Llamas J, Barrera J, Solano J. Tratamiento temprano de Clase III. Rev Esp 2011; $41: 79-89$. 15.- Romero B, Estrada A. Máscara facial de Protracción como tratamiento de Maloclusiones Clase III - Reporte de Caso 
Clínico. Revista Latinoamericana de Ortodoncia y Odontopediatría 2010. Obtenible en https://www.ortodoncia.ws/publicaciones/2010/art30.as

16.- Tashima A, Verrastro A. Tratamento Ortodôntico Precoce da Mordida Cruzada Anterior e Posterior: Relato de Caso Clínico Early Orthodontic Treatment of Anterior and Posterior Crossbite: Case Report. J Bras Odontopediatr Odontol Bebê, 2003; 6 (29): 24-31.

Recibido: 21-01-2016

Acrptado: 28-08-2016

Correspondencia: Johana Maldonado-Villamizar.

joha_maldonado22@yahoo.com.mx

Urb. El Tulipán. Parcela 24. Torre D. Apto D-13. +58 4244472331 